\title{
Circulating Calreticulin Is Increased in Myelofibrosis: Correlation with Interleukin-6 Plasma Levels, Bone Marrow Fibrosis, and Splenomegaly
}

\author{
Daria Sollazzo, Dorian Forte, Nicola Polverelli, Margherita Perricone, Marco Romano, \\ Simona Luatti, Nicola Vianelli, Michele Cavo, Francesca Palandri, and Lucia Catani
}

Department of Experimental, Diagnostic and Specialty Medicine, Institute of Hematology "L. and A. Seràgnoli", University of Bologna, Bologna, Italy

Correspondence should be addressed to Lucia Catani; lucia.catani@unibo.it

Received 13 April 2016; Accepted 17 August 2016

Academic Editor: Tomoki Nakamura

Copyright (C) 2016 Daria Sollazzo et al. This is an open access article distributed under the Creative Commons Attribution License, which permits unrestricted use, distribution, and reproduction in any medium, provided the original work is properly cited.

\begin{abstract}
Myelofibrosis (MF) is a clonal neoplasia of the hemopoietic stem/progenitor cells associated with genetic mutations in the Janus kinase 2 (JAK2), myeloproliferative leukemia virus oncogene (MPL), and calreticulin (CALR) genes. MF is also characterized by a state of chronic inflammation. Calreticulin (CRT), as a multifunctional protein, is involved in a spectrum of cellular processes including inflammation, autoimmunity, and cancer initiation/progression. Based on this background, we hypothesised that in MF circulating CRT might reflect the inflammatory process. In the present study we show that circulating CRT is increased in MF patients compared to healthy controls. Also, in MF, CRT levels highly correlate with bone marrow fibrosis, splenomegaly, and Interleukin-6 (IL-6) plasma levels. In turn, higher IL-6 levels also correlated with disease severity in terms of increased spleen size, bone marrow fibrosis, number of circulating $\mathrm{CD}_{3} 4^{+}$cells, and lower hemoglobin values. These results demonstrate that the circulating CRT takes part in the inflammatory network of MF and correlates with aggressiveness of the disease.
\end{abstract}

\section{Introduction}

Myelofibrosis (MF) is a Philadelphia-negative myeloproliferative neoplasm (Ph-neg MPN) that may arise de novo (Primary Myelofibrosis, PMF) or after Essential Thrombocythemia (ET; PET-MF) and Polycythemia Vera (PV; PPV$\mathrm{MF})$. MF is a rare blood cancer with an incidence of about 0.58 new cases per 100.000 people per year but with higher prevalence because of a chronic and disabling course leading always to death due to progression and disease-related or treatment-related complications. It is a clonal disorder of the hemopoietic stem/progenitor cells which is clinically characterized by worsening constitutional symptoms, progressive splenomegaly, bone marrow (BM) fibrosis, and cytopenias as well as by an increased risk to develop thrombotic complications and acute leukemia [1-3].

Driver mutations in Janus kinase 2 (JAK2), calreticulin $(C A L R)$, and myeloproliferative leukemia virus oncogene $(M P L)$ have been reported. However, less than $10 \%$ of patients have nonmutated JAK2, MPL, and CALR genes ("triplenegative"). Regardless of molecular status, all patients have dysregulation in the JAK/STAT signaling [2-6].

Together with molecular abnormalities, MF is characterized by abnormal expression of several proinflammatory and immunoregulating cytokines secreted by activated leukocytes and platelets/megakaryocytes. This inflammatory microenvironment has emerged as a key player in MF pathogenesis [712].

Physiologically, CRT was first described as an endoplasmic reticulum protein responsible for $\mathrm{Ca}^{2+}$ homeostasis and glycoprotein folding; currently, CRT is recognized as a multifunctional chaperone detected in other cellular compartments, as well as extracellularly, where it is involved in cell proliferation, phagocytosis, apoptosis, adhesion, and innate and adaptive immune processes including cancer cell elimination by immunogenic cell death and fibrosis [13]. CRT overexpression is linked to various pathological conditions including chronic inflammatory diseases, autoimmunity, 
fibrosis-related disorders, and malignant evolution [14-18]. In MF, the mutated CRT protein was found to constitutively activate the MPL receptor signaling [19, 20].

Given the CRT involvement in inflammation, fibrosis, and cancer, we hypothesised that in MF circulating CRT might reflect the inflammatory process. Here, we characterized the circulating CRT levels of MF patients. Moreover, we investigated the correlation between CRT levels and various clinical and laboratory parameters.

\section{Materials and Methods}

2.1. Study Population. Peripheral blood (PB) was obtained from 30 patients with MF in chronic phase and from 10 healthy age-matched volunteers. The diagnosis of MF was made according to WHO 2008 criteria [21]. Patients and controls provided written informed consent for the study. This study was approved by the medical Ethical Committee of the University Hospital of Bologna and was conducted in accordance with the Declaration of Helsinki.

2.2. Assay of Circulating Proteins. Here we analyzed the plasma levels of CRT in patients/controls. EDTA-anticoagulated PB was centrifuged for 15 minutes at $1000 \times \mathrm{g}$ within 30 minutes of collection. The plasma was then collected and stored at $-80^{\circ} \mathrm{C}$ until quantification. CRT was evaluated by a commercially available ELISA assay (Cusabio Biotech Co., Wuhan, China), according to the manufacturer's instructions. Briefly, a standard curve of $100 \mu \mathrm{L}$ aliquots of known concentrations of recombinant CRT was run and triplicate $100 \mu \mathrm{L}$ samples were added to the wells. CRT binding was detected using a biotin/avidin system. The plates were then assessed by ELISA on a plate reader at $450 \mathrm{~nm}$. The Ciraplex ${ }^{\mathrm{TM}}$ immunoassay kit/Human 9-Plex Array (Aushon BioSystems, Billerica, MA, USA) was used for the measurement of circulating IL- 6 and TNF- $\alpha$.

2.3. Molecular Pattern. Molecular analyses were assessed at diagnosis or before treatment's start on DNA obtained from granulocytes. Driver mutations were analyzed as previously described [22]. Specifically, $J A K 2^{\mathrm{V} 617 \mathrm{~F}}$ mutation was evaluated with ipsogen JAK2 MutaQuant Kit. The percentage of mutant $J A K 2^{\mathrm{V} 617 \mathrm{~F}}$ allele was expressed as the ratio of $J A K 2^{\mathrm{V} 617 \mathrm{~F}}$ copies to total copy number $(\mathrm{CN})$ of $J A K 2(\mathrm{CN}$ of $J A K 2^{\mathrm{V} 617 \mathrm{~F}}+\mathrm{CN}$ of $J A K 2$ wild type). CALR exon 9 sequencing was performed by Next Generation Sequencing (NGS) approach with GS Junior (Roche-454 platform); analysis was carried out with AVA Software (GRCh38 as referenced). Rare CALR mutations identified by NGS were confirmed by Sanger sequencing. $M P L$ mutations were investigated by ipsogen $M P L W 515 K / L$ MutaScreen Kit and by Sanger sequencing (for MPLS505N and other secondary exon 10 mutations).

2.4. Cytogenetic Analysis. Chromosome banding analysis was performed on BM cells by standard banding techniques according to the International System for Human Cytogenetic Nomenclature [23]. At least 20 metaphases were required. Unfavorable karyotype was defined according to the Dynamic International Prognostic Score System (DIPSS) plus [24] and included complex karyotype or single or two abnormalities including $+8,-7 / 7 q^{-}, \mathrm{i}(17 \mathrm{q}),-5 \% 5 \mathrm{q}-, 12 \mathrm{p}-$, $\operatorname{inv}(3)$, or $11 \mathrm{q} 23$ rearrangement.

2.5. Statistical Analysis. Statistical analyses (Wilcoxon test and Spearman correlation analysis) were performed using GraphPad (GraphPad Software Inc., La Jolla, CA). All $p$ values were considered statistically significant when $p \leq 0.05$ (two-tailed).

\section{Results}

A total of $30 \mathrm{MF}$ patients were investigated: $J A K 2^{V 617 F}-(16$ cases), CALR- (10 cases), and MPL- (3 cases) mutated. One patient was triple-negative. $C A L R$-mutated patients were type 1 (8 cases) and type 2 (2 cases). Patients characteristics are shown in Table 1 . Fifteen patients were at diagnosis. Thirteen patients received previous therapies for MF (hydroxyurea (10 cases) and ruxolitinib (3 cases)); however, at the time of the study, they were untreated for at least 2 months.

As shown in Figure 1(a), we found significantly higher CRT plasma levels in MF patients as compared with healthy subjects (median, $5.2 \mathrm{ng} / \mathrm{mL}$, and range, 1.4-25, versus median, $1.8 \mathrm{ng} / \mathrm{mL}$, and range, $1.2-3.7 ; p=0.0028)$. Comparing CRT plasma levels of $J A K 2^{V 617 F}$ and CALRmutated patients, no significant differences were observed (Figure 1(b)). Even though few patients were studied, CRT plasma levels of MPL-mutated and triple-negative patients were superimposable to the other MF patients (Figure 1(b)). CRT plasma levels of patients at diagnosis were not significantly different from those of the other patients. No correlation was observed between circulating CRT levels and hemoglobin levels, white blood cells/platelets count, and circulating $\mathrm{CD} 34^{+}$cells number.

Along with CRT plasma levels, circulating TNF- $\alpha$ (median: $2.62 \mathrm{pg} / \mathrm{mL}$; range: $0.05-9.37$ ) and IL-6 (median: $33.3 \mathrm{pg} / \mathrm{mL}$; range: 8.7-258.9) were also increased in MF patients as compared to healthy subjects (median, $0.26 \mathrm{pg} / \mathrm{mL}$, and range, $0-0.84$, and median, $6.37 \mathrm{pg} / \mathrm{mL}$, and range, $4.5-32.8$, resp.; $p=0.008$ ) (Figures $1(\mathrm{c})$ and $1(\mathrm{~d})$ ). TNF- $\alpha$ and IL- 6 plasma levels were not affected by mutational status and allele burden (data not shown). Interestingly, in MF, irrespective of patients being at diagnosis or not, there was a positive correlation between the plasma levels of CRT and BM fibrosis $(p=0.038 ; r=0.39)$, splenomegaly $(p=0.0089 ; r=0.47)$, and circulating IL-6 $(p=0.028$; $r=0.42$ ) (Figures 2(a), 2(b), and 2(c)). This correlation was also irrespective of mutational status (comparing $J A K 2^{V 617 F}$. mutated and CALR-mutated patients). In turn, IL-6 plasma levels correlated with BM fibrosis $(p=0.0056 ; r=0.49)$, splenomegaly $(p=0.018 ; r=0.46)$, and the number of circulating CD $34^{+}$cells $(p=0.029 ; r=0.48)$ and correlated negatively with hemoglobin values $(p=0.047 ; r=-0.39$; Figures 3(a), 3(b), 3(c), and 3(d)).

\section{Discussion}

There has been a lack of understanding regarding the role of soluble CRT in MF. The first result of the study is 


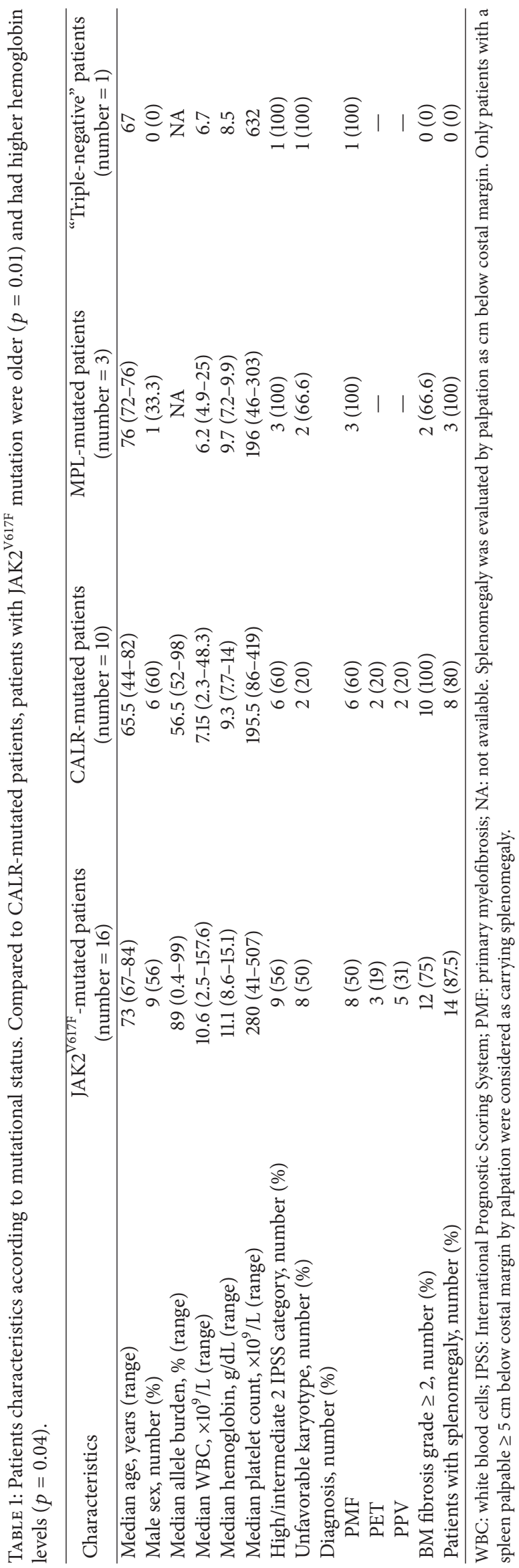




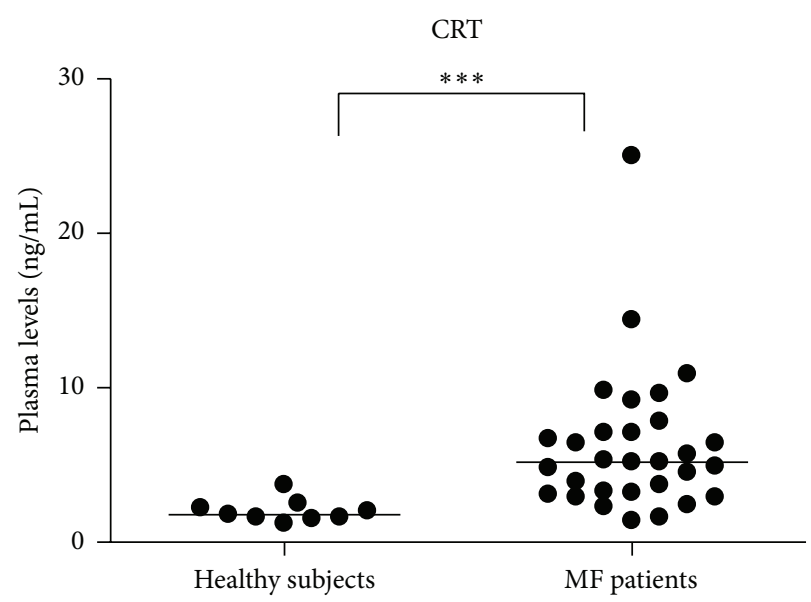

(a)

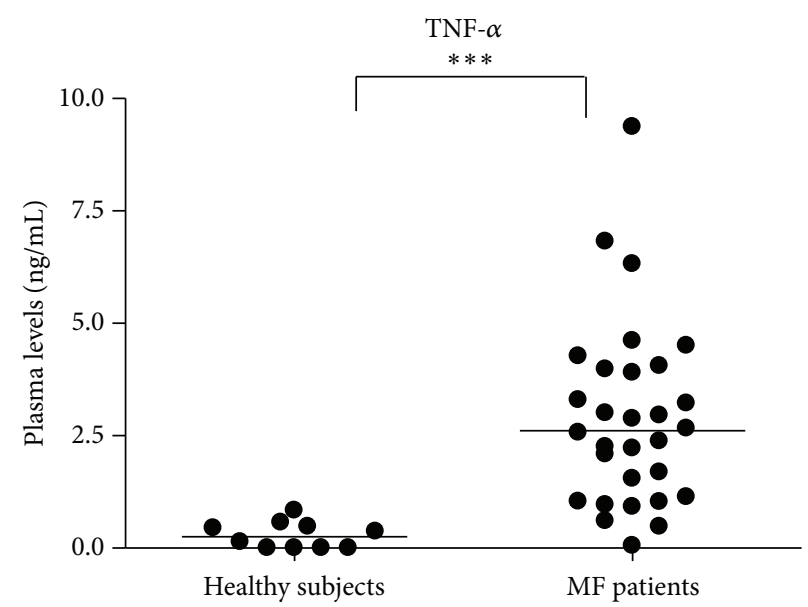

(c)

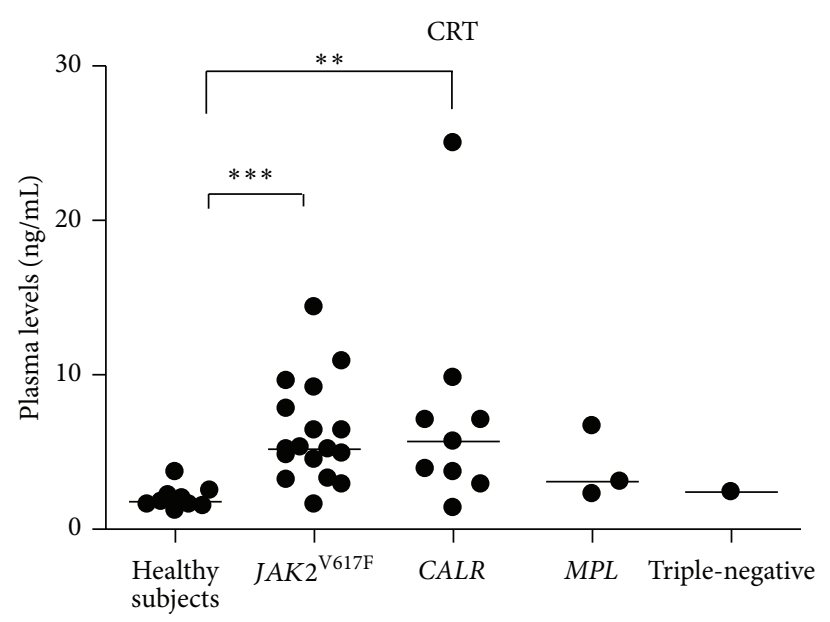

(b)

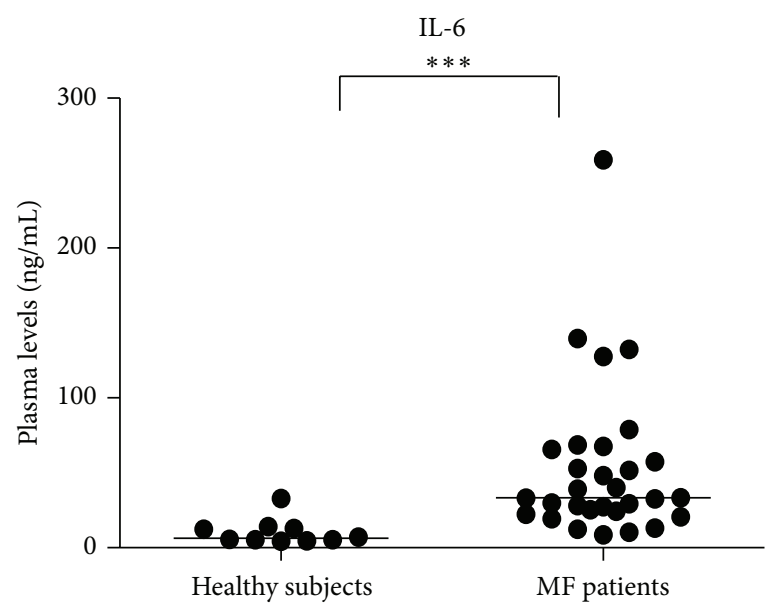

(d)

FIGURE 1: Analysis of the circulating levels of CRT, IL-6, and TNF- $\alpha$ proteins. The CRT plasma levels of total MF (a) or MF subdivided into $J A K 2^{V 617 F}$-mutated $(n=16), C A L R$-mutated $(n=10), M P L$-mutated $(n=3)$, and triple-negative-mutated $(n=1)$ groups $(\mathrm{b})$ were measured by ELISA. Compared with age-matched controls (HD; $n=10)$, CRT plasma levels were significantly increased in MF patients $(p=0.0028)$. Of note, there was no significant difference between the mutated groups. Irrespective of mutational status, TNF- $\alpha$ (c) and IL-6 (d) blood plasma levels were also increased in MF $(p=0.008)$. For all graphs, one symbol represents one individual, and the height of the bar represents the median value. ${ }^{* *} p \leq 0.01 ;{ }^{* * *} p \leq 0.001$.

that in MF CRT plasma levels are increased compared to healthy controls. CRT has been found to have a preferential expression in megakaryocyte/platelets either from normal subjects or from patients with Ph-neg MPN (and regardless of mutation status) [25]. Therefore, these cells, which show abnormal number/function in MF, are likely to be the major contributors to the augmented amount of circulating CRT. Previous studies support the hypothesis that extracellular and soluble CRT is mainly released from dead, dying, or inflamed/stressed cells [13-16]. Consequently, the high CRT levels detected in MF may primarily be due to the chronic inflammatory state that characterizes both the marrow and peripheral niches and reflect impairment in tissue homeostasis.

The second result is that CRT plasma levels are equally increased in $\mathrm{JAK} 2^{\mathrm{V} 617 \mathrm{~F}}$-mutated and CALR-mutated $\mathrm{MF}$ patients. In this study, we used an antibody that is directed against the $\mathrm{N}$ terminus of CRT and is expected to label both mutated and unmutated proteins. Therefore, the circulating protein that was detected in CALR-positive patients is likely to be the sum of mutated (hemopoietic restricted) and unmutated molecules. This datum may suggest that the acquisition of mutations in the CALR gene, although causing the hyperactivation of the MPL receptor $[19,20]$, does not induce an increased circulating CRT amount.

Herein, we therefore demonstrated that CRT protein levels were found to directly correlate with the clinical aggressiveness of the disease in terms of larger spleen size and more severe marrow fibrosis. In addition, we found a direct correlation between circulating plasma levels of CRT and IL6 , one of the most potent proinflammatory cytokines which is upregulated in MF [26]. In turn, higher IL-6 levels also correlated with disease severity in terms of increased spleen size, marrow fibrosis, number of circulating $\mathrm{CD}^{+} 4^{+}$cells, 


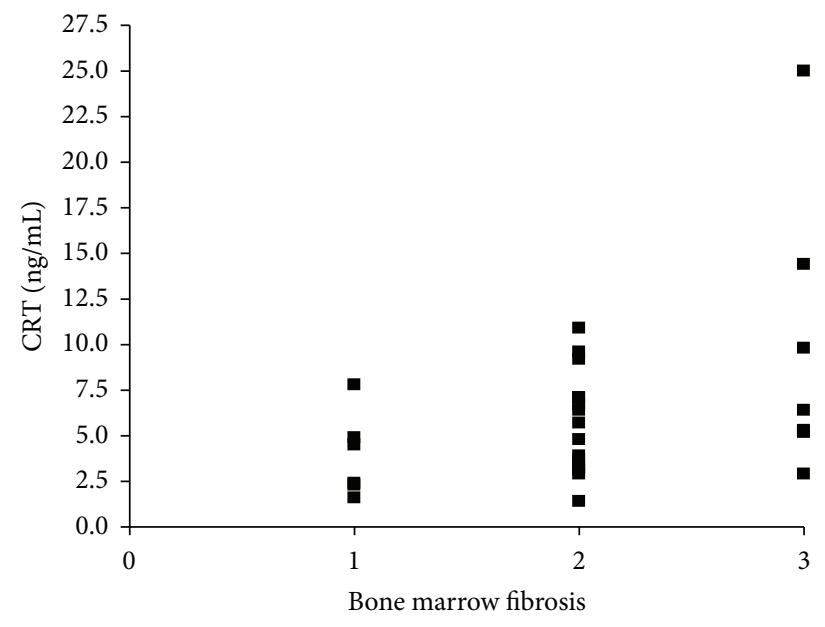

(a)

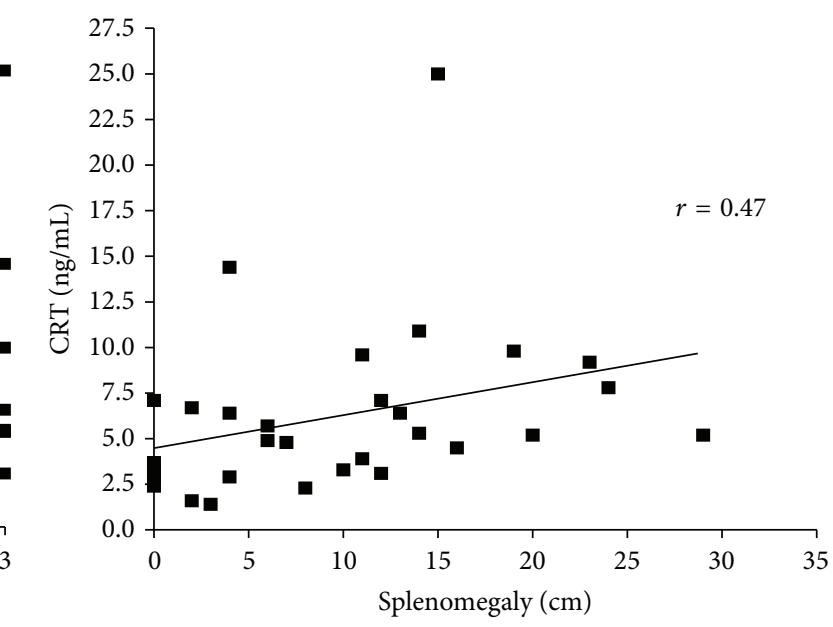

(b)

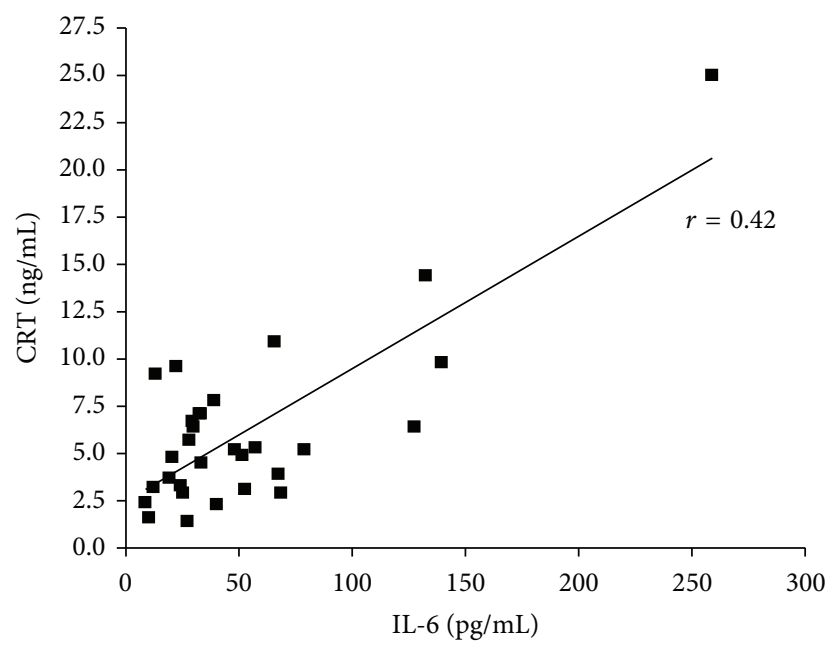

(c)

FIgURE 2: Correlation between CRT plasma levels and BM fibrosis, splenomegaly, and circulating IL-6. Circulating CRT positively correlates with fibrosis, splenomegaly, and soluble IL-6 in MF. Scatterplots demonstrating correlation between the plasma levels of CRT and BM fibrosis ( $p=0.038 ; r=0.39)$, splenomegaly $(p=0.0089 ; r=0.47)$, and circulating IL-6 $(p=0.028 ; r=0.42)$ in MF patients $(\mathrm{a}, \mathrm{b}$, and $\mathrm{c}$, resp.) are shown. $x$-axis of (a) shows BM fibrosis scale.

and lower hemoglobin values. Even though these correlations were weak ( $r$ always below 0.5), all together point out the involvement of CRT in the inflammatory network and in disease aggressiveness. The correspondence between CRT and IL- 6 plasma levels may be at least partially justified by the recent discovery that soluble CRT induces active mRNA transcription through MAPK and NF-kB activation in macrophages, thereby augmenting their IL- 6 and TNF$\alpha$ production [27]. In addition, recently, conditioned media from cells expressing type I mutant CALR have been shown to exaggerate cytokine production from normal monocytes [28]. It is therefore likely that in MF the increased circulating CRT may contribute to the disease-related inflammation/fibrosis through positively enhancing IL- 6 production. By contrast, despite the fact that TNF- $\alpha$ is a negative regulator of CRT expression [29], no correlation was observed between circulating CRT and TNF- $\alpha$ plasma levels in our MF patients, suggesting the presence of a TNF- $\alpha$-independent mechanism of regulation.
Taken together, our data highlight the role of this protein in the inflammatory network of MF. A mutual interaction among CRT and other inflammatory cytokines including IL6 may indeed contribute to the generation/maintenance of inflammation/fibrosis of MF.

Potential limitation of the present study is the small sample size of patients. Nonetheless, our data create the rational basis for future studies investigating the role of circulating CRT in the inflammatory network of MF and other Phnegative MPNs in larger cohorts of patients. Notably, due to correlation with fibrosis and splenomegaly, circulating CRT measurement may be useful in clinical practice.

\section{Conclusion}

We conclude that the elevated plasma CRT levels in MF patients parallel the degree of disease activity and inflammatory state. This may identify patients with more severe disease who might benefit from tailored therapy. 


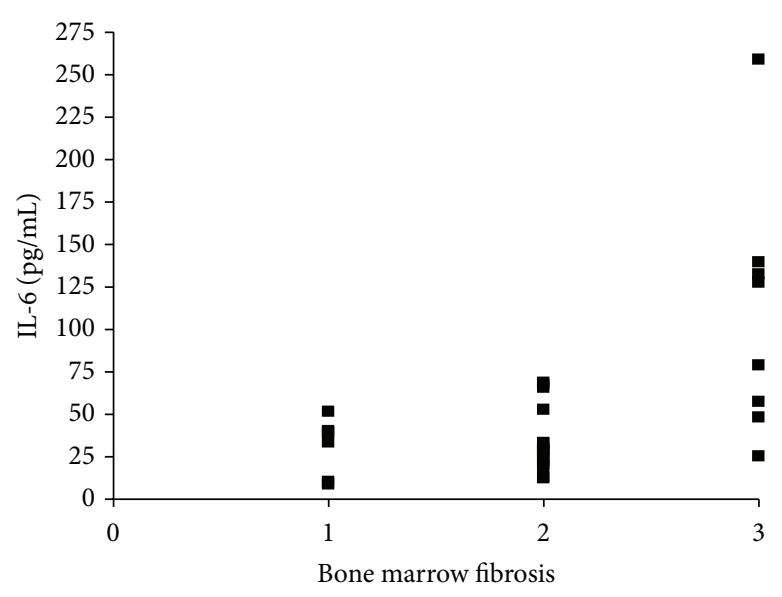

(a)

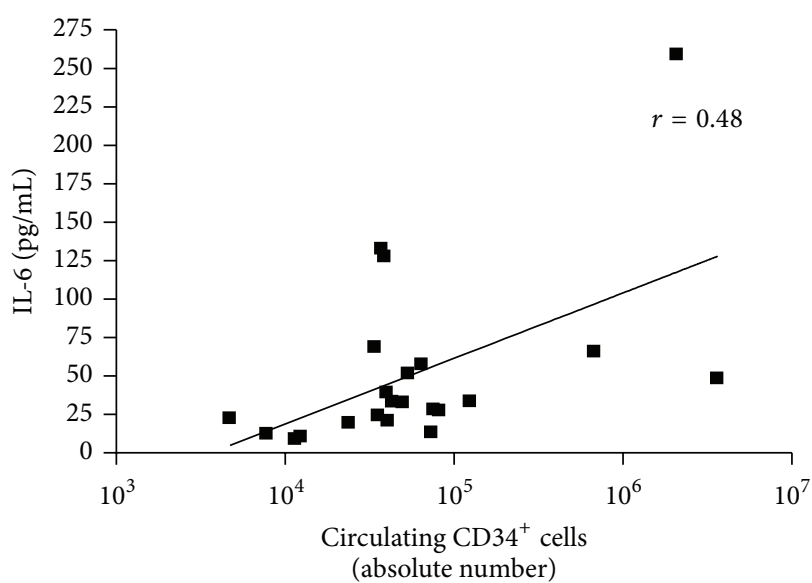

(c)

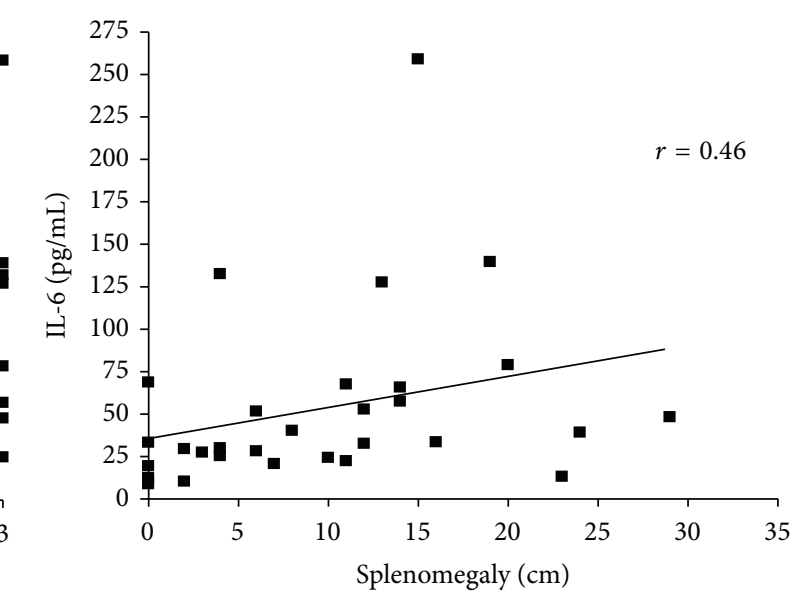

(b)

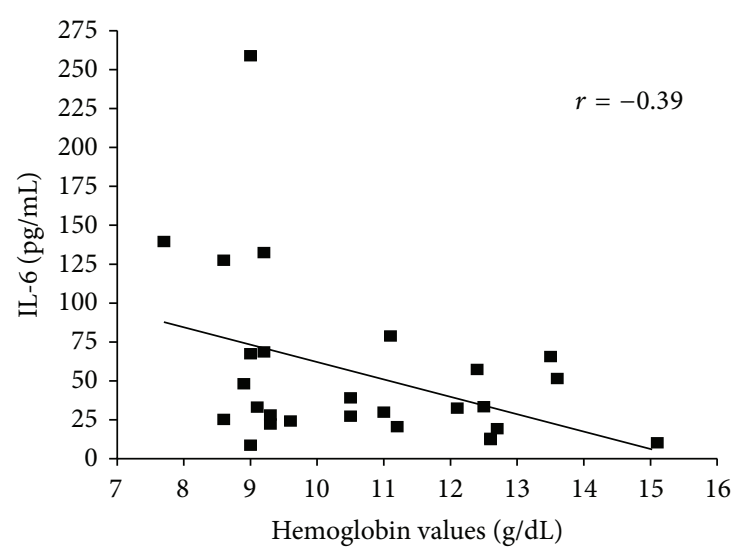

(d)

FIGURE 3: Correlation between IL-6 plasma levels and BM fibrosis, splenomegaly, number of circulating CD34 ${ }^{+}$cells, and hemoglobin values. Irrespective of mutational status, IL-6 plasma levels correlated with BM fibrosis $(p=0.0056 ; r=0.49)$, splenomegaly $(p=0.018 ; r=0.46)$, and the absolute number of circulating CD34 ${ }^{+}$cells $(p=0.029 ; r=0.48)$ and negatively correlated with hemoglobin values $(p=0.047$; $r=-0.39$ ); (a, b, c, and d, resp.). $x$-axis of (a) shows BM fibrosis scale.

\section{Competing Interests}

The authors declare that they have no competing interests.

\section{Authors' Contributions}

Daria Sollazzo and Lucia Catani designed the study; Nicola Polverelli, Nicola Vianelli, and Francesca Palandri recruited the patients and coordinated their samples collection; Daria Sollazzo, Dorian Forte, Margherita Perricone, and Marco Romano performed the laboratory work and analyzed data; Nicola Vianelli and Michele Cavo supervised the study; Daria Sollazzo, Lucia Catani, and Francesca Palandri wrote the manuscript. Daria Sollazzo, Dorian Forte, Francesca Palandri, and Lucia Catani contributed equally to this work.

\section{Acknowledgments}

This research was supported by Associazione Italiana contro le Leucemie-Bologna (BolognAIL) and by the University of Bologna (RFO 2014 for Lucia Catani and Francesca Palandri).

\section{References}

[1] M. Kleppe and R. L. Levine, "New pieces of a puzzle: the current biological picture of MPN," Biochimica et Biophysica ActaReviews on Cancer, vol. 1826, no. 2, pp. 415-422, 2012.

[2] M. Cazzola and R. Kralovics, "From Janus kinase 2 m Janus kinase 2 to calreticulin: the clinically relevant genomic landscape of myeloprolife," Blood, vol. 123, no. 24, pp. 3714-3719, 2014.

[3] A. Tefferi and A. Pardanani, "Myeloproliferative neoplasms: a contemporary review," JAMA Oncology, vol. 1, no. 1, pp. 97-105, 2015.

[4] T. Klampfl, H. Gisslinger, A. S. Harutyunyan et al., "Somatic mutations of calreticulin in myeloproliferative neoplasms," The New England Journal of Medicine, vol. 369, no. 25, pp. 23792390, 2013. 
[5] J. Nangalia, C. E. Massie, E. J. Baxter et al., "Somatic CALR mutations in myeloproliferative neoplasms with nonmutated JAK2," New England Journal of Medicine, vol. 369, no. 25, pp. 2391-2405, 2013.

[6] R. Rampal, F. Al-Shahrour, O. Abdel-Wahab et al., "Integrated genomic analysis illustrates the central role of JAK-STAT pathway activation in myeloproliferative neoplasm pathogenesis," Blood, vol. 123, no. 22, pp. e123-e133, 2014.

[7] H. C. Hasselbalch, "The role of cytokines in the initiation and progression of myelofibrosis," Cytokine and Growth Factor Reviews, vol. 24, no. 2, pp. 133-145, 2013.

[8] A. G. Fleischman, "Inflammation as a driver of clonal evolution in myeloproliferative neoplasm," Mediators of Inflammation, vol. 2015, Article ID 606819, 6 pages, 2015.

[9] H. L. Geyer, A. C. Dueck, R. M. Scherber, and R. A. Mesa, "Impact of inflammation on myeloproliferative neoplasm symptom development," Mediators of Inflammation, vol. 2015, Article ID 284706, 9 pages, 2015.

[10] H. C. Hasselbalch and M. E. Bjørn, "MPNs as inflammatory diseases: the evidence, consequences, and perspectives," Mediators of Inflammation, vol. 2015, Article ID 102476, 16 pages, 2015.

[11] S. Hermouet, E. Bigot-Corbel, and B. Gardie, "Pathogenesis of myeloproliferative neoplasms: role and mechanisms of chronic inflammation," Mediators of Inflammation, vol. 2015, Article ID 145293, 16 pages, 2015.

[12] A. Tefferi, R. Vaidya, D. Caramazza, C. Finke, T. Lasho, and A. Pardanani, "Circulating interleukin (IL)-8, IL-2R, IL-12, and IL15 levels are independently prognostic in primary myelofibrosis: a comprehensive cytokine profiling study," Journal of Clinical Oncology, vol. 29, no. 10, pp. 1356-1363, 2011.

[13] W.-A. Wang, J. Groenendyk, and M. Michalak, "Calreticulin signaling in health and disease," International Journal of Biochemistry and Cell Biology, vol. 44, no. 6, pp. 842-846, 2012.

[14] S. E. Pike, L. Yao, J. Setsuda et al., "Calreticulin and calreticulin fragments are endothelial cell inhibitors that suppress tumor growth," Blood, vol. 94, no. 7, pp. 2461-2468, 1999.

[15] W.-F. Cheng, C.-F. Hung, C.-Y. Chai et al., "Tumor-specific immunity and antiangiogenesis generated by a DNA vaccine encoding calreticulin linked to a tumor antigen," The Journal of Clinical Investigation, vol. 108, no. 5, pp. 669-678, 2001.

[16] L. Gold, D. Williams, J. Groenendyk, M. Michalak, and P. Eggleton, "Unfolding the complexities of ER chaperones in health and disease: report on the 11th international calreticulin workshop," Cell Stress and Chaperones, vol. 20, no. 6, pp. 875883, 2015

[17] Y.-C. Lu, W.-C. Weng, and H. Lee, "Functional roles of calreticulin in cancer biology," BioMed Research International, vol. 2015, Article ID 526524, 9 pages, 2015.

[18] P. Eggleton, E. Bremer, E. Dudek, and M. Michalak, "Calreticulin, a therapeutic target?" Expert Opinion on Therapeutic Targets, vol. 20, no. 9, pp. 1137-1147, 2016.

[19] I. Chachoua, C. Pecquet, M. El-Khoury et al., "Thrombopoietin receptor activation by myeloproliferative neoplasm associated calreticulin mutants," Blood, vol. 127, no. 10, pp. 1325-1335, 2016.

[20] M. Araki, Y. Yang, N. Masubuchi et al., "Activation of the thrombopoietin receptor by mutant calreticulin in CALRmutant myeloproliferative neoplasms," Blood, vol. 127, no. 10, pp. 1307-1316, 2016.

[21] J. W. Vardiman, J. Thiele, D. A. Arber et al., "The 2008 revision of the World Health Organization (WHO) classification of myeloid neoplasms and acute leukemia: rationale and important changes," Blood, vol. 114, no. 5, pp. 937-951, 2009.
[22] F. Palandri, R. Latagliata, N. Polverelli et al., "Mutations and long-term outcome of 217 young patients with essential thrombocythemia or early primary myelofibrosis," Leukemia, vol. 29, no. 6, pp. 1344-1349, 2015.

[23] “ISCN 1995," in Guidelines for Cancer Cytogenetics, Supplement to: An International System for Human Cytogenetic Nomenclature, F. Mitelman, Ed., S Karger, Basel, Switzerland, 1995.

[24] F. Passamonti, F. Cervantes, A. M. Vannucchi et al., "A dynamic prognostic model to predict survival in primary myelofibrosis: a study by the IWG-MRT (International Working Group for Myeloproliferative Neoplasms Research and Treatment)," Blood, vol. 115, no. 9, pp. 1703-1708, 2010.

[25] A. M. Vannucchi, G. Rotunno, N. Bartalucci et al., "Calreticulin mutation-specific immunostaining in myeloproliferative neoplasms: pathogenetic insight and diagnostic value," Leukemia, vol. 28, no. 9, pp. 1811-1818, 2014.

[26] K. E. Panteli, E. C. Hatzimichael, P. K. Bouranta et al., "Serum interleukin (IL)-1, IL-2, sIL-2Ra, IL-6 and thrombopoietin levels in patients with chronic myeloproliferative diseases," British Journal of Haematology, vol. 130, no. 5, pp. 709-715, 2005.

[27] C.-C. Duo, F.-Y. Gong, X.-Y. He et al., "Soluble calreticulin induces tumor necrosis factor- $\alpha$ (TNF- $\alpha$ ) and interleukin (IL)- 6 production by macrophages through mitogen-activated protein kinase (MAPK) and $\mathrm{NF \kappa B}$ signaling pathways," International Journal of Molecular Sciences, vol. 15, no. 2, pp. 2916-2928, 2014.

[28] M. R. Garbati, C. A. Welgan, S. H. Landefeld et al., "Mutant calreticulin-expressing cells induce monocyte hyperreactivity through a paracrine mechanism," American Journal of Hematology, vol. 91, no. 2, pp. 211-219, 2016.

[29] S. Vig, A. K. Pandey, G. Verma, and M. Datta, "C/EBP $\alpha$ mediates the transcriptional suppression of human calreticulin gene expression by TNF $\alpha$," The International Journal of Biochemistry \& Cell Biology, vol. 44, no. 1, pp. 113-122, 2012. 


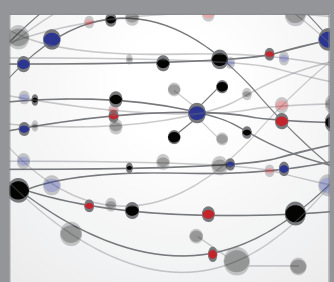

The Scientific World Journal
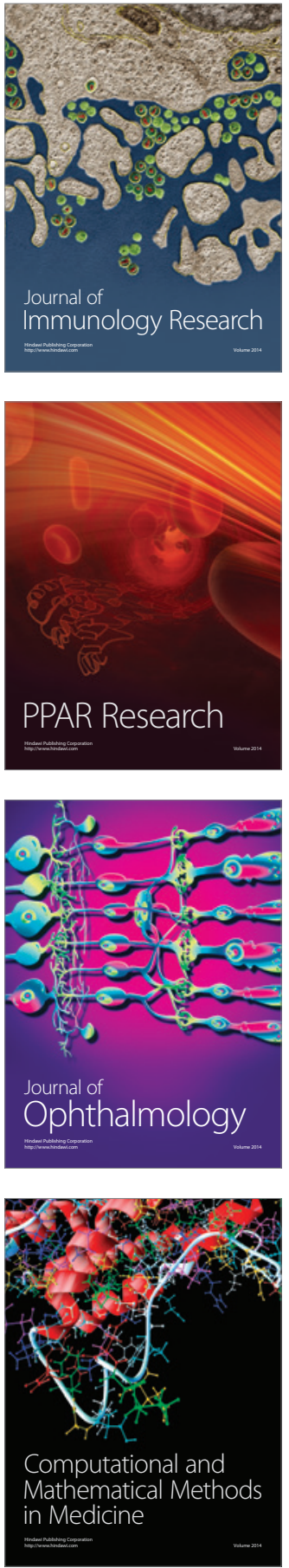

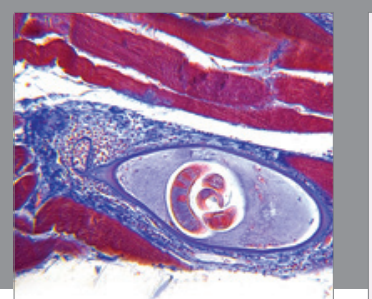

Gastroenterology Research and Practice

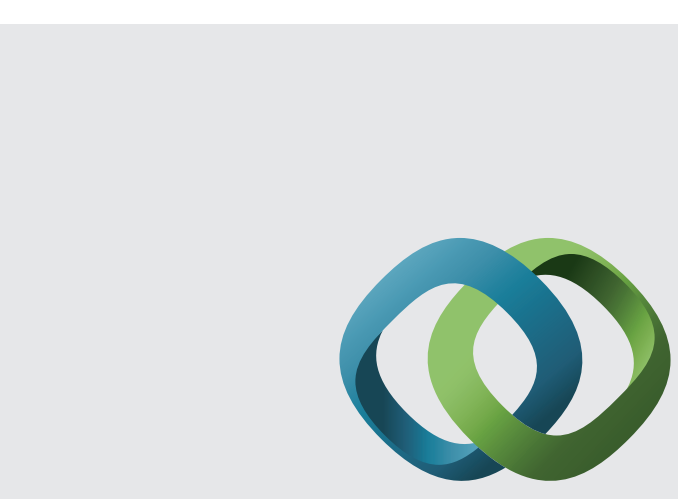

\section{Hindawi}

Submit your manuscripts at

http://www.hindawi.com
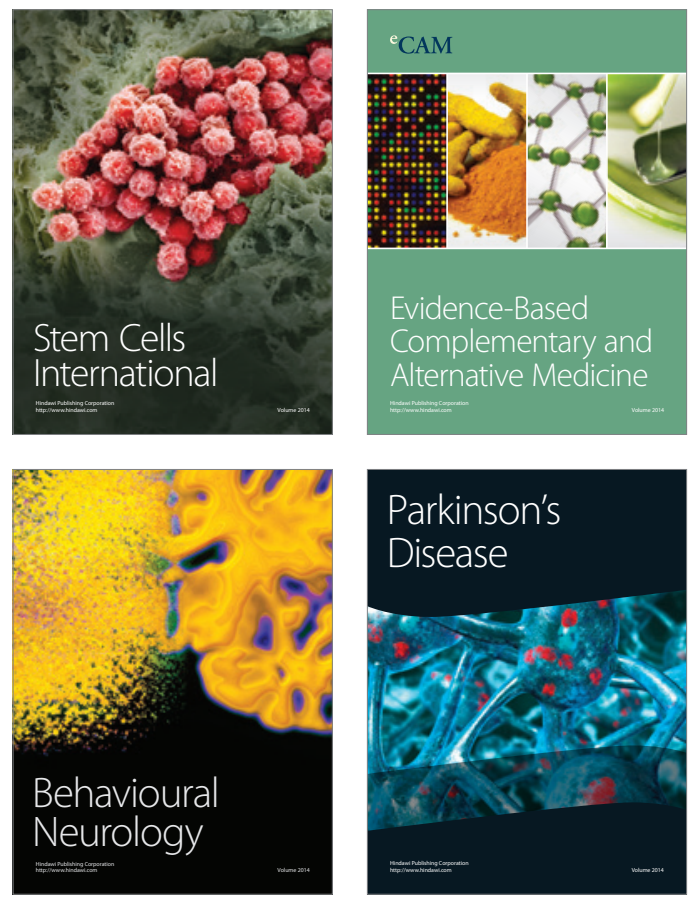
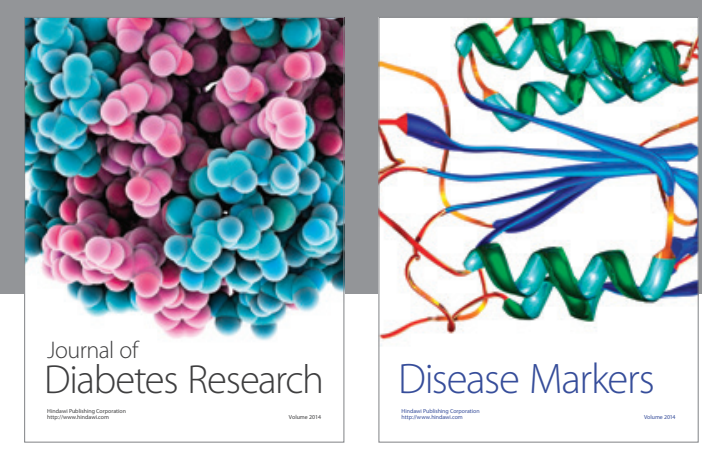

Disease Markers
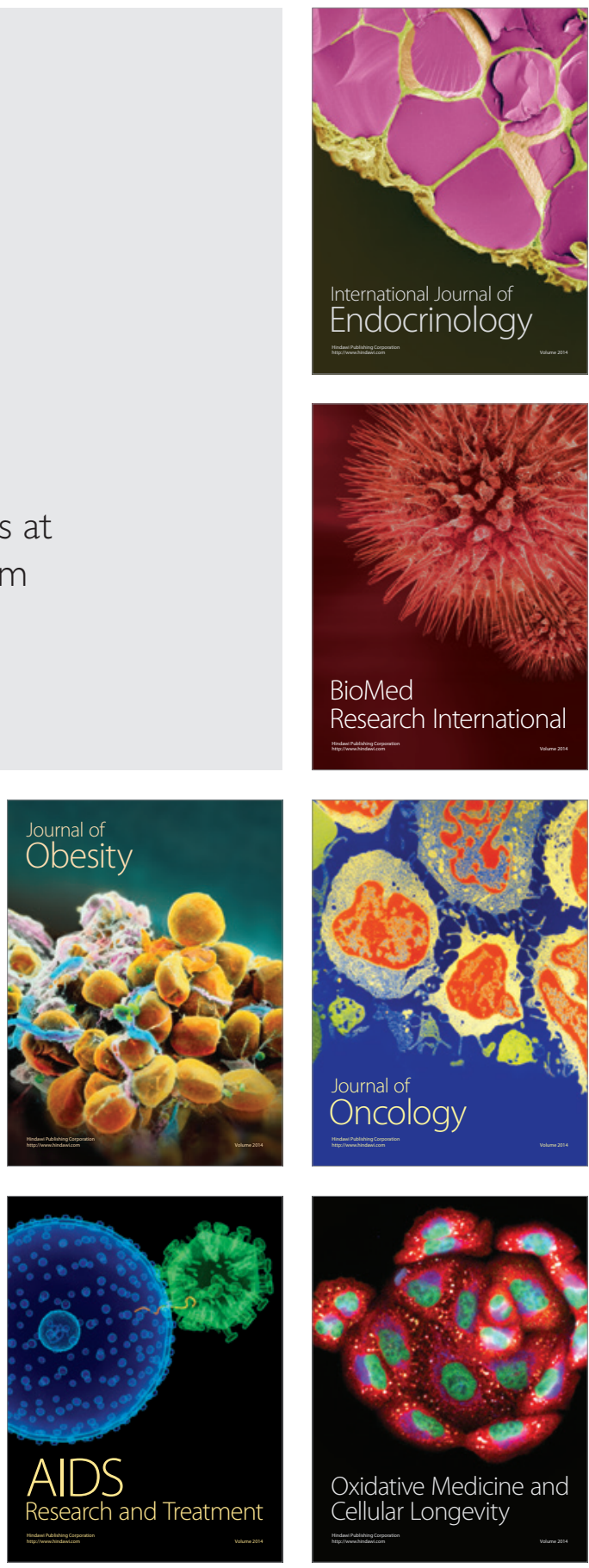